\title{
Conditioned heart-rate change in the pigeon: Analysis and prediction of acquisition patterns
}

\author{
DAVID H. COHEN and DENNIS M. GOFF \\ Department of Physiology, University of Virginia School of Medicine, Charlottesville, Virginia 22901
}

\begin{abstract}
A large sample of pigeons $(n=458)$ was trained in a classical conditioning paradigm where whole-field retinal illumination and footshock were paired to produce conditioned heart-rate change. Analysis of the acquisition data indicated two distinct learning patterns (denoted $\mathrm{C}$ and C), and any given group of animals can be characterized by describing the proportion of animals showing each acquisition pattern (the $\mathrm{C}: \overline{\mathrm{C}}$ ratio). It is shown that mean learning curve variability among groups is largely a function of group differences in this ratio, such that accurate prediction of mean learning curves is possible knowing only this parameter. Furthermore, this ratio is itself predictable only from responses to an initial visual stimulus presentation. Consequently, from reactivity (orienting response) to a single presentation of the to-be-conditioned stimulus, it is possible to predict accurately the mean learning curve of any given group.
\end{abstract}

Some years ago, we initiated a program to develop visually conditioned heart-rate change in the pigeon as a vertebrate model system for cellular studies of long-term, associative learning (Cohen, 1969, 1974a, in press). Numerous studies in the intervening years have generated a large sample of animals trained in a standardized paradigm where whole-field retinal illumination is paired with footshock to establish conditioned cardioacceleration. We have collated the data of 458 animals trained under such standardized conditions from 1969 through 1975, and we present an analysis of these data with a view toward describing (1) annual and seasonal variation, (2) the detailed properties of response acquisition, and (3) different patterns of conditioned response development. The rationale of this analysis was to gain further understanding of conditioned heart-rate change, first by determining the contributions of such factors as season and baseline heart rate. More importantly, however, we hoped to determine if there are different "classes" of animals with respect to acquisition pattern and if this information would allow prediction of conditioning performance. This, in turn, would provide a firmer foundation for relating cellular neurophysiological and conditioning data.

\section{CONDITIONING PARADIGM}

\section{Training Procedures}

The apparatus and general training procedures have been previously described (e.g., Cohen, 1975;

This research was supported by National Science Foundation Grants BMS-74-22258 and BNS-75-20537 and a grant from the Scottish Rite Foundation. D. H. Cohen was partially supported by P. H. S. Research Career Development Award HL-16579 from the National Heart and Lung Institute.
Cohen \& Durkovic, 1966; Cohen \& Pitts, 1968). To summarize, the birds were semirestrained and placed in a dark conditioning chamber. The conditioned stimulus, a 6 -sec, $100-\mathrm{fL}$ light, was presented on a panel at the front of the chamber. The unconditioned stimulus, an 0.5 -sec footshock $(60-\mathrm{Hz}$, ac), was delivered immediately upon termination of this light and was adjusted to maintain the unconditioned response in the range of $350-450$ beats $/ \mathrm{min}$; this generally required currents of $0.2-1.5 \mathrm{~mA}$. Training was accomplished over 3 days with 40 light-shock pairings on each of the first 2 days and 20 pairings on the 3rd day. The same intertrial interval schedule was used throughout, having a mean of $3.7 \mathrm{~min}$ and a range of 2.5-5.0 min (Cohen \& Macdonald, 1971).

\section{Response Analysis}

To analyze heart-rate changes, baseline heart rate (BASE) was calculated by counting the number of $R$ waves in the 6 -sec control period just preceding light onset and expressing this number in beats/min. A similar count of $\mathbf{R}$ waves during the light period gave the absolute conditioned response (CR) in beats/min, and subtracting the baseline heart rate from this absolute conditioned response then gave the rate change during the conditioned stimulus presentation (CR-BASE). For more detailed analysis of the conditioned response dynamics, the 6-sec light periods were divided into 1 -sec intervals and the number of $\mathbf{R}$ waves counted in each interval. This permitted estimation of the rates for successive $1-\mathrm{sec}$ intervals of the conditioned stimulus period and, when compared with baseline heart rate, gave the conditioned heart rate change for each interval. These various measures, when averaged over trials and/or animals, are equivalent in resolution to using $R-R$ interval measures (Cohen \& Pitts, 1968). 


\section{Robustness of the Paradigm}

While the data described here are from a standardized paradigm, it should be pointed out that conditioned heart-rate change is readily established under many variations of this paradigm. For example, response dynamics and acquisition rate are unaffected by such variables as degree of restraint (Cohen \& Macdonald, 1971), pre-presentations of the conditioned stimulus (Cohen \& Macdonald, 1971), and pharmacological blockade of the neuromuscular junction (Migani-Wall, Cabot, \& Cohen, 1977; Gold $\&$ Cohen, Note 2). It has been shown that prepresentation of a large number of unpaired lights and shocks can transiently retard acquisition (Cohen \& Macdonald, 1971); short mean intertrial intervals can produce a trial-massing effect (Cohen \& Macdonald, 1971), and nonphysiological conditioned stimulus intensities can increase the sensitization component of performance (Cohen, 1974b). However, conditioned heart-rate change can still be established and remains monotonic cardioacceleration. Moreover, similar responses develop with conditioned stimuli in other modalities such as audition (e.g., Tuge \& Yueh, 1962), and this same general paradigm is effective for establishing differential responding (e.g., Cohen \& Durkovic, 1966). Finally, once developed, the conditioned heart-rate change is highly resistant to extinction (Cohen, 1967; Cohen \& Durkovic, 1966). Thus, a robust conditioned heartrate change is readily established in the pigeon under a variety of training conditions, and, while response magnitude and acquisition rate may vary as a function of training conditions, with shock as the unconditioned stimulus the response is invariably monotonic cardioacceleration.

\section{Role of Orienting and Sensitization Responses}

It is important next to consider nonassociative responses that occur during application of our basic paradigm. These include unconditioned heart-rate change to light, which we refer to as the orienting response, and sensitization responses to light, which are a function of noncontingent shock occurrence. With respect to the orienting response, we have consistently reported (e.g., Cohen, 1975; Cohen \& Durkovic, 1966; Cohen \& Macdonald, 1971) a small cardioacceleration during the initial light presentation prior to its association with footshock. That this orienting response is visually evoked is confirmed by its absence in bilaterally enucleated animals (Cohen, Note 1). The dynamics of this response resemble those of the conditioned response in being monotonic cardioacceleration (Figure 1). However, we have shown that, either with repeated light presentations not followed by shock (Cohen \& Macdonald, 1971), or with lumbar dorsal rhizotomies preventing transmission of the unconditioned stimulus information
(Leonard \& Cohen, 1975), this orienting response rapidly habituates (Figure 1).

Sensitization responses have been studied on various occasions with unpaired lights and shocks (Cohen, 1974b; Cohen \& Durkovic, 1966; Cohen \& Macdonald, 1971, Cohen \& Pitts, 1968; Migani-Wall et al., 1977; Gold \& Cohen, Note 2). The results consistently show only a small sensitization response with distinctly different dynamics from orienting and conditioned responses. These latter responses are monotonic cardioacceleration, while the sensitization response is an early acceleration followed by a deceleration and subsequent acceleration (Figure 1). Moreover, analogous to habituation of the orienting response, sensitized heart-rate change also habituates within approximately 10 trials (Figure 1).

Thus, after approximately 10 conditioning trials, heart-rate responses are free of contributions from nonassociative responses. In contrast, during the initial 10 trials, responses would seem to be a joint function of orienting, sensitization, and early conditioning. However, this may not be the case, since Cohen (1974a) has shown that responses during these initial trials are equivalent in animals with previous extinction of sensitization and animals given systematic light-shock pairings. This would imply that in some manner the early conditioned response is preemptive when training begins with systematic lightshock pairings.

\section{Heart Rate Change as a Primary Response}

A final prefatory issue regards possible interactions of heart rate with concomitantly developing conditioned responses. In most, if not all, conditioning situations, the conditioned stimulus elicits an integrated response pattern, and in our paradigm heart-rate change is but one component of a pattern
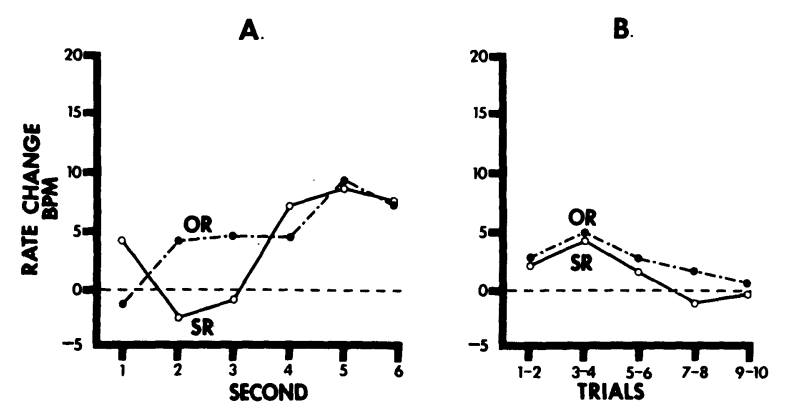

Figure 1. Panel A illustrates the response dynamics for orienting (OR) and sensitization (SR) responses. The OR group received lights only on the standardized intertrial schedule. The SR group received unpaired lights and footshocks. The curves show mean heart-rate changes from baseline for 1-sec intervals of the light presentation. Panel $B$ illustrates the habituation of these responses. The curves show mean heart-rate changes between the stimulus period and a preceding 6-sec control period averaged over two-trial blocks. BPM $=$ beats $/ \mathrm{min}$ (from Cohen, 1974a). 
Table 1

Distribution of Sample Over Years and Months

\begin{tabular}{crrrrrrrrrrrrrr}
\hline Year & Jan & Feb & March & April & May & June & July & August & Sept & Oct & Nov & Dec & Year N \\
\hline 1969 & 3 & 3 & 1 & 2 & 1 & 2 & 8 & 5 & 4 & 4 & 4 & 6 & 43 \\
1970 & 4 & 4 & 4 & 4 & 3 & 5 & 4 & 2 & 5 & 3 & 5 & 6 & 49 \\
1971 & 9 & 0 & 0 & 0 & 17 & 16 & 5 & 7 & 8 & 8 & 8 & 4 & 82 \\
1972 & 6 & 7 & 6 & 6 & 10 & 6 & 7 & 6 & 8 & 9 & 9 & 8 & 88 \\
1973 & 8 & 8 & 8 & 9 & 8 & 6 & 0 & 7 & 6 & 10 & 8 & 5 & 83 \\
1974 & 9 & 8 & 7 & 7 & 2 & 5 & 9 & 4 & 6 & 3 & 1 & 6 & 67 \\
1975 & 7 & 4 & 4 & 5 & 3 & 4 & 4 & 3 & 4 & 1 & 4 & 3 & 46 \\
Month N & 46 & 34 & 30 & 33 & 44 & 44 & 37 & 34 & 41 & 38 & 39 & 38 & 458 \\
\hline
\end{tabular}

which includes changes in respiratory rate and volume (e.g., Cohen \& Durkovic, 1966), arterial blood pressure, and a variety of somatic responses (Cohen, 1969). These concomitant responses could evoke afferent neural activity which feeds back to modify or even generate the heart-rate response, an issue of concern in the heart-rate conditioning literature for some years and recently treated at length for our specific paradigm (Cohen, 1974c).

To review briefly, we can exclude contributions from conditioned changes in circulating levels of hormones having chronotropic effects, since elimination of the cardiac innervation precludes conditioned heart-rate change (Cohen \& Pitts, 1968). Contributions from respiratory responses and muscular activity can also be excluded, since we have shown that eliminating these responses by neuromuscular blockade does not alter conditioning performance (Migani-Wall et al., 1977; Gold \& Cohen, Note 2). Finally, preliminary data indicate only a slight increase in mean arterial pressure concomitant with conditioned cardioacceleration. This small pressor response is attenuated or eliminated when conditioned cardiac changes are prevented by $\beta$-adrenergic blockade, suggesting that it is secondary to increased cardiac output and results only minimally from conditioned changes in peripheral resistance. Thus, evidence to date clearly indicates that heart-rate change is not secondary to concomitant conditioned responses, and that such responses do not even obviously affect the heart-rate response. The heart-rate change should perhaps be viewed as a major component of a conditioned cardiovascular response pattern, including increased cardiac output and possibly redistribution of blood to muscle beds. Such a pattern would provide the cardiovascular adjustments required by the somatic responses of a conditioned defense reaction (Cohen \& Obrist, 1975).

\section{SAMPLE CHARACTERISTICS}

All data were obtained from male and femalt White Carneaux pigeons (Columba livia) obtained from the Palmetto Pigeon Plant, Sumter, South Carolina. The birds weighed $400-700 \mathrm{~g}$ (mean $=552 \mathrm{~g}$ ) and ranged in age from 2-6 months. All were experimentally naive at the start of training, were individually housed with free access to food and water, and remained on a 12-h light-dark cycle for at least 10 days before training. The sample consisted of 458 birds trained between January 1969 and December 1975 (Table 1). It was composed of control animals from various lesion studies, and each animal had thus undergone a sham operation 10-14 days prior to training. In this procedure, the birds were anesthetized with sodium pentobarbital, a midline incision made, the periosteum reflected, and the wound sutured. All birds were then trained in the standardized, 3-day paradigm described above.

\section{ANNUAL AND SEASONAL VARIATION}

In this section, we describe variation among years and months in baseline heart-rate and conditioning performance. For these analyses, mean values for the 100 training trials were calculated for baseline rate (BASE), absolute conditioned response (CR), and conditioned rate change (CR-BASE)-see Response Analysis. With respect to variation among years, baseline heart rate and the two measures of conditioning performance are shown in Figure 2A for each year. Analysis of variance indicated significant variation in baseline rates among years, $F(6,451)=14.60$, $\mathrm{p}<.001$. However, Duncan's multiple range test suggested that this resulted from the unusually high rates in 1974, $\mathrm{p}<.005$, and we cannot explain the perturbation for this single year. Heart rates during conditioned stimulus periods also varied significantly among years, $F(6,451)=20.56, p<.001$, and again Duncan's multiple range test indicated that this variability was due to the 1974 scores, $p<.005$. As seen in Figure 2A, heart rates during control and conditioned stimulus periods are positively correlated, $r_{s}=+.78, p<.05$, and thus the elevated conditioned response levels in 1974 probably reflect the high baseline rates in that year. Since conditioned heart-rate change (CR-BASE) tends to dissociate conditioned response and baseline levels, it was not elevated in 1974 (Figure 2A). However, there was still significant variation in CR-BASE scores among 

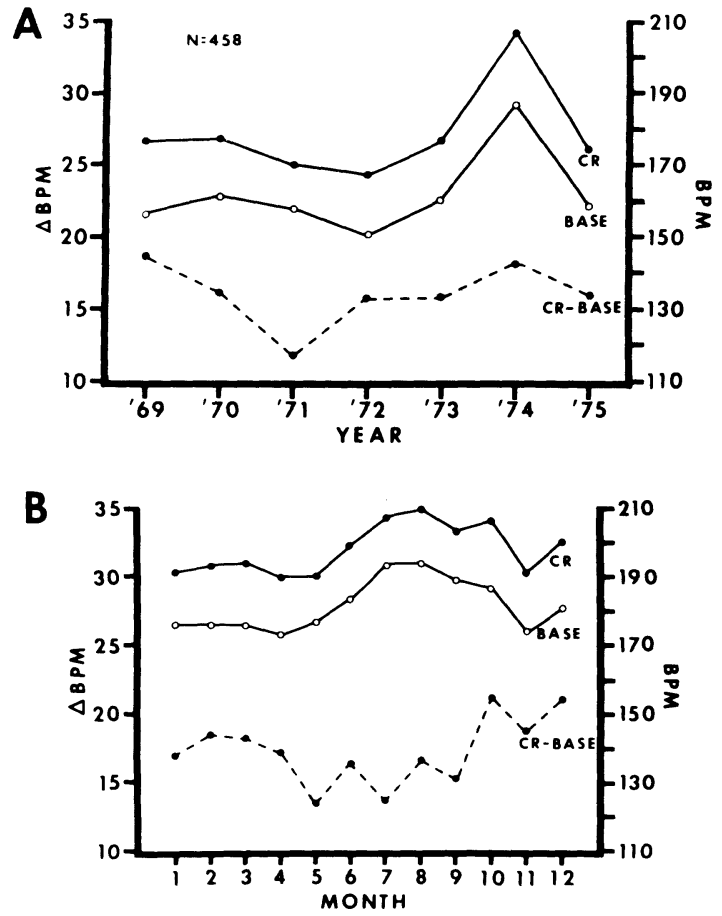

Figure 2. Panel A illustrates yearly variation from 1969 ('69) to 1975 ('75) in baseline heart rate (BASE), heart rate during the conditioned stimulus period (CR), and the rate difference between conditioned stimulus and baseline periods (CR-BASE). $B A S E$ and $C R$ are referred to the right ordinate where BPM $=$ beats $/ \mathrm{min}$. CR-BASE is referred to the left ordinate where $\triangle B P M=$ the rate change from baseline in beats $/ \mathrm{min}$. Each point represents a 100 -trial mean for the birds trained that year. (See text for further explanation of BASE, CR, and CRBASE.) Panel B illustrates monthly variation (January $=1$ through December $=12$ ) in BASE, CR, and CR-BASE scores. BASE and CR are referred to the right ordinate and CR-BASE to the left. Each point represents a 100-trial mean for the birds trained that month, irrespective of year.

years, $F(6,451)=3.93, \mathrm{p}<.005$, but in this case Duncan's multiple range test indicated that the low 1971 scores accounted for the variability, $\mathrm{p}<.005$. The 1971 data can perhaps be explained by sampling, since in $197165 \%$ of the animals were trained in the 5-month period from May through September (Table 1). Conditioned heart-rate change tends to be reduced in this period, and therefore, with more distributed seasonal sampling in 1971, annual variation in CR-BASE scores might not have been statistically significant.

Given no obvious annual trends, the yearly samples were pooled and analyzed for monthly variation (Table 1, Figure 2B). Regarding baseline, there was significant seasonal variation, $F(11,446)=29.66$, $\mathrm{p}<.001$, with heart rates rising in May through August and then declining; such monthly variation is not surprising in view of well-documented seasonal changes in many avian physiological functions. Since conditioned heart rate parallels baseline, $\mathrm{r}_{\mathrm{s}}=+.79$, $\mathrm{p}<.01$, it also varied among months, $\mathrm{F}(11,446)=$ $83.13, \mathrm{p}<.001$. That this seasonal change in conditioning is attributable to baseline variation is further suggested by the absence of significant variation in CR-BASE scores, despite their tendency to fall during elevated baseline periods (Figure 2B).

\section{TOTAL SAMPLE ANALYSIS OF PERFORMANCE}

As with years, pooling the data over months also seemed justified, provided analyses are restricted to the CR-BASE measure of conditioning performance. This measure does not significantly correlate with baseline heart rate, and, because it largely dissociates conditioned response and baseline levels, seasonal variation is minimized. Consequently, in this section we describe acquisition results for the entire sample of 458 birds, using CR-BASE scores as the measure of conditioning performance.

\section{Baseline Heart Rate}

Baseline heart rates for the initial 10 trials and for subsequent 10-trial blocks are shown in Figure 3, and analysis of the 10-trial block data indicated significant variation over training, $F(9,4570)=15.67$, $\mathrm{p}<.001$. From inspection of Figure 3, it appears that this variation results from elevated baseline rates at the start of each training day with subsequent within-session declines; this is a persistent pattern with no apparent differences among sessions. Baseline rates also varied significantly within the first 10 trials, $\mathrm{F}(9,4570)=6.79, \mathrm{p}<.001$, increasing from Trial 1 to Trial 2 and then declining with a plateau between Trials 5 and 6 (Figure 3). This may in part be a function of intertrial interval, since the shortest interval ( $2.5 \mathrm{~min}$ ) occurs between Trials 1 and 2 and between Trials 5 and 6 . However, the occurrence of the first scheduled shock presentation between 1 and 2 is also

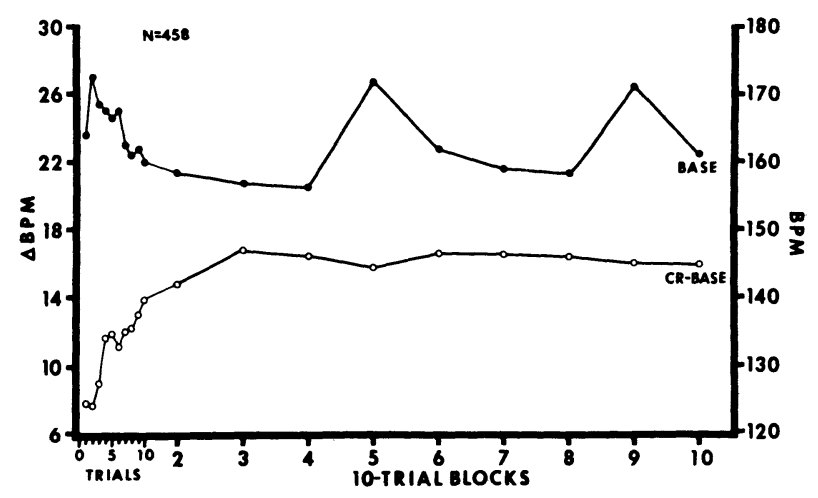

Figure 3. Mean baseline rates (BASE) and mean acquisition curves (CR-BASE) of the total sample for each of the initial 10 trials and for subsequent 10-trial blocks. BASE is referred to the right ordinate (BPM) and CR-BASE to the left ordinate ( $\triangle B P M)$. 

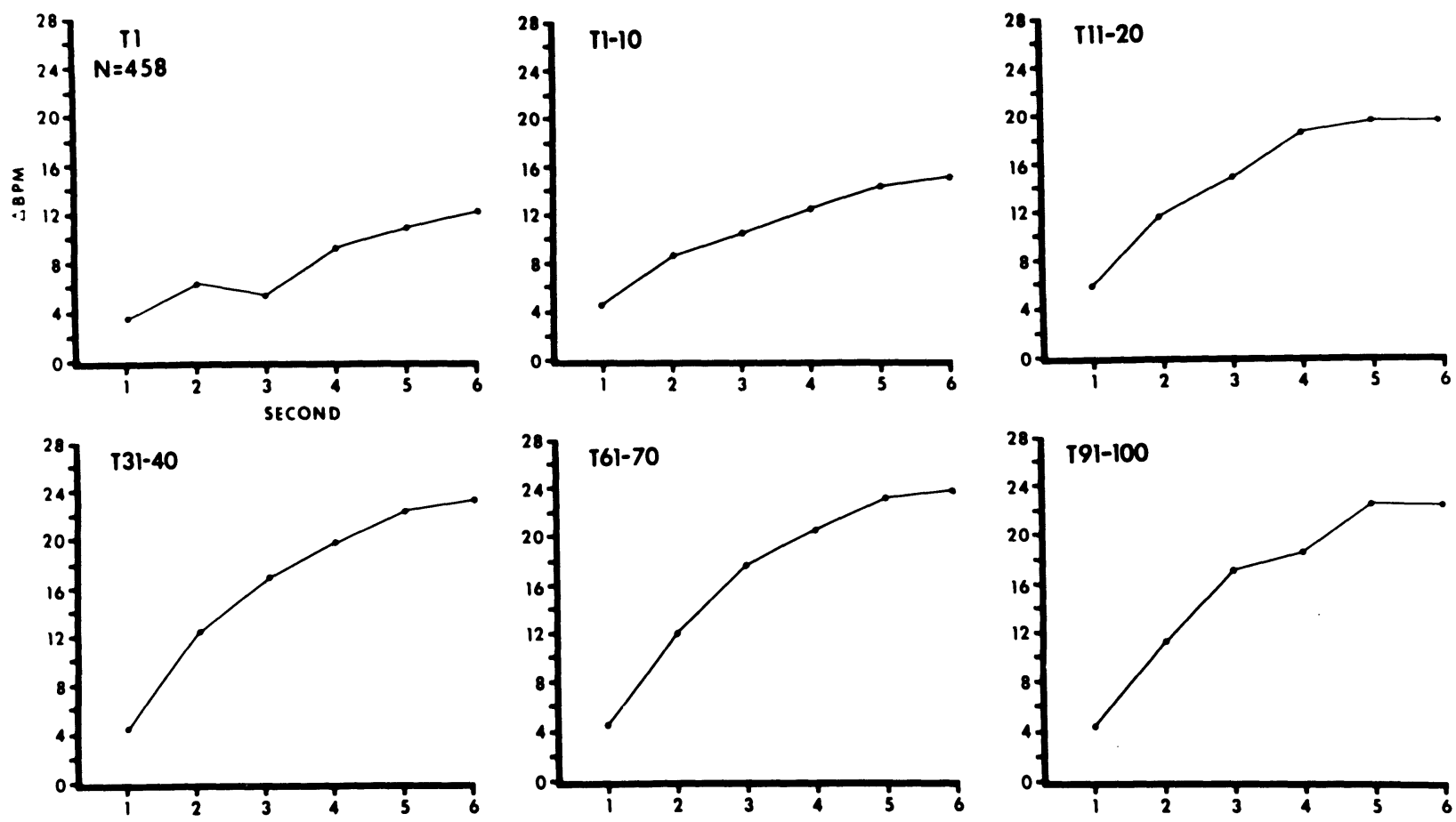

Figure 4. Mean response dynamics of the total sample for Trial 1 (T1) and for the 1st (T1-10), 2nd (T11-20), 4th (T31-40), 7th (T61-70), and 10th (T91-100) trial blocks. The curves show mean heart-rate change from baseline ( $\triangle \mathrm{BPM})$ for 1-sec intervals of the conditioned stimulus period.

likely to contribute significantly to the large rate increase between these trials.

\section{Orienting Response}

The heart-rate response on the initial training trial provided a measure of the orienting response to the light. As seen in Figure 3, the mean response was a rate increase of 7.8 beats $/ \mathrm{min}$, which compares favorably with the previously reported mean of 9.0 beats/min (Cohen \& Macdonald, 1971). The dynamics of this response are shown in Figure 4, and $t$ tests indicated that heart rate in each of the six 1-sec intervals differed significantly from baseline. While this conflicts with our earlier report (Cohen \& Macdonald, 1971), where heart rate did not differ significantly until the third second, a later section of this paper dealing with individual differences in response patterns resolves this discrepancy.

\section{Conditioned Response Development}

As illustrated in Figure 3, the heart-rate response grows rapidly over the first 10 trials, such that by the 10th trial a mean increase of 13.8 beats/min occurs. This is achieved by a steady growth in response magnitude with perturbations on Trials 2 and 6 (Figure 3). Again, we would point out that these trials are preceded by the shortest intertrial interval that may produce a trial-massing effect (Cohen \& Macdonald, 1971). The response dynamics for this initial training block are illustrated in Figure 4. While analysis of variance indicated a significant increase in response magnitude over these trials, $F(9,27420)=16.32$, $\mathrm{p}<.001$, there was no significant Trials by Dynamics interaction. Thus, monotonic rate increases of similar slope occur on each trial, but they become displaced successively higher on the ordinate as training progresses, with peak responses of $\cong 19$ beats $/ \mathrm{min}$ on the 10th trial.

Analysis of variance of the 10-trial block data indicated significant response magnitude increases over training, $F(9,27420)=8.01, p<.001$. This was accounted for by changes in the first two blocks, and separate analysis of subsequent blocks showed no significant changes from the third block onward (Figure 3). It might also be noted that the standard errors of these 10-trial means are small and consistent over training; for example, for Blocks 1, 2, 4, 7, and 10 , these errors were $\pm .47, .53, .54, .35$, and .53 beats/min, respectively. Regarding response dynamics (Figure 4), they too remained unchanged over these later blocks, and $t$ tests indicated that significant rate increases occurred in each of the six 1 -sec intervals of each block.

Thus, the total sample results suggest a steady growth in response magnitude during the initial 20 or 30 training trials where the mean conditioned response reaches an asymptote and remains stable over the remaining trial blocks. Variability of these mean 
responses is small and does not change systematically over training. Similarly, after the first few trials, response latency is $<1 \mathrm{sec}$ and remains so throughout training.

\section{PATTERNS OF RESPONSE ACQUISITION}

\section{Establishing Criterion Performance}

While the above analysis demonstrates a smooth, negatively accelerated learning curve, such mean curves can obscure significant individual differences. Therefore, a criterion of asymptotic performance was established and applied to individual conditioning performances. For each animal, a moving response mean for each 10-trial block was calculated using CR-BASE scores. The block with the largest mean was identified, and a response range of $\pm 20 \%$ around this value was established. Criterion was then defined as the first 10-trial block in which mean performance fell within this range, with the means of all subsequent blocks remaining in that range. This criterion was applied to each of the 458 birds, and the results are summarized in Figure 5. An unexpected finding was that $100(21.8 \%)$ birds never achieved criterion, and they were thus defined as the noncriterion or $\overline{\mathrm{C}}$ group; the remaining 358 birds were defined as the criterion or $\mathrm{C}$ group.

\section{Comparison of Criterion and \\ Noncriterion Acquisition}

The mean learning curves of these two groups are shown in Figure 6, and they indicate clear differences in the pattern of response acquisition. The $\mathrm{C}$ group acquired the response with a smooth, negatively

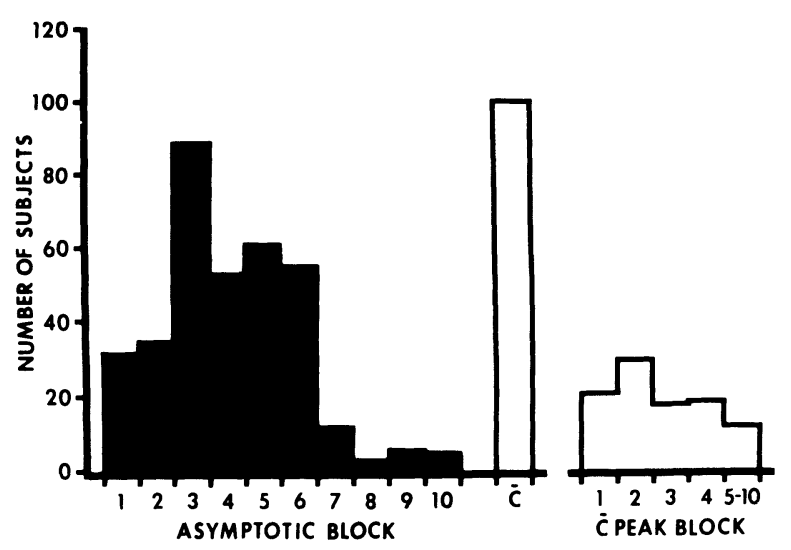

Figure 5. Histogram illustrating the distribution of birds with respect to the asymptotic criterion measure (see text). The solid bars of the left histogram show the animals achieving this criterion ( $\mathrm{C}$ group) and their distribution with respect to the 10-trial block in which asymptote was reached. The open bar of the left histogram indicates animals not reaching criterion $(\bar{C}$ group), and their distribution with respect to the 10-trial block where the peak response occurred is shown in the histogram at the right.

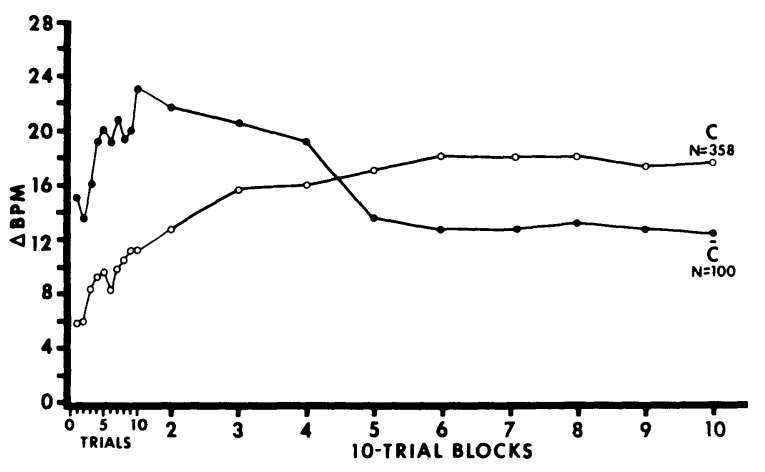

Figure 6. Mean acquisition curves for animals of the criterion (C) and noncriterion $(\bar{C})$ groups.

accelerated learning curve similar to the mean curve of the total sample (Figure 3). However, $\bar{C}$ animals reached peak performance in early training and then declined to a lower plateau for later blocks. Analysis of variance of the 10-trial block data indicated no significant difference in overall response levels of the $\mathrm{C}$ and $\overline{\mathrm{C}}$ groups, $\mathrm{F}(1,456)<1$, but the Groups by Blocks interaction was significant, $F(9,4104)=19.29$, $p<.001$. These results are understandable from inspection of Figure 6 , where $\overline{\mathrm{C}}$ performance exceeds $C$ response levels in early blocks and then declines such that the overall group means do not differ.

To explore differences between these groups in more detail, we analyzed the dynamics of the 6-sec responses, beginning with the orienting response. As seen in Figure 7, the $\bar{C}$ group had a larger orienting response, $F(1,456)=7.93, p<.001$, and the Groups by Dynamics interaction was also significant, $\mathrm{F}(5,2280)=49.51, \mathrm{p}<.001$. In the $\bar{C}$ group, cardioacceleration significantly differed from baseline, beginning with the $1 \mathrm{st} \mathrm{sec}$, while the $\mathrm{C}$ group did not respond significantly in Seconds 1 and 3. The orienting response dynamics of the $\mathrm{C}$ group are quite similar to those' described previously (Cohen \& Macdonald, 1971), perhaps suggesting that our earlier. sample included considerably more $C$ than $\bar{C}$ birds. In any case, $C$ and $\bar{C}$ groups are discriminable even before training begins, since the orienting response of the $\bar{C}$ group has shorter latency and is of higher magnitude.

Regarding performance during the initial 10 lightshock pairings, both groups showed clear response development, $\mathrm{F}(9,4104)=17.11, \mathrm{p}<.001$ (Figure 6). However, the Groups by Trials interaction was not significant, such that the difference in overall response magnitude of the groups was maintained, $F(1,456)=$ $84.82, \mathrm{p}<.001$. Analyses of variance were then performed over these 10 trials for each of the 1-sec intervals of the stimulus period, and the $\bar{C}$ group responded at significantly higher levels in all intervals: $\mathrm{p}<.001$ for all comparisons (Figure 7). Furthermore, 

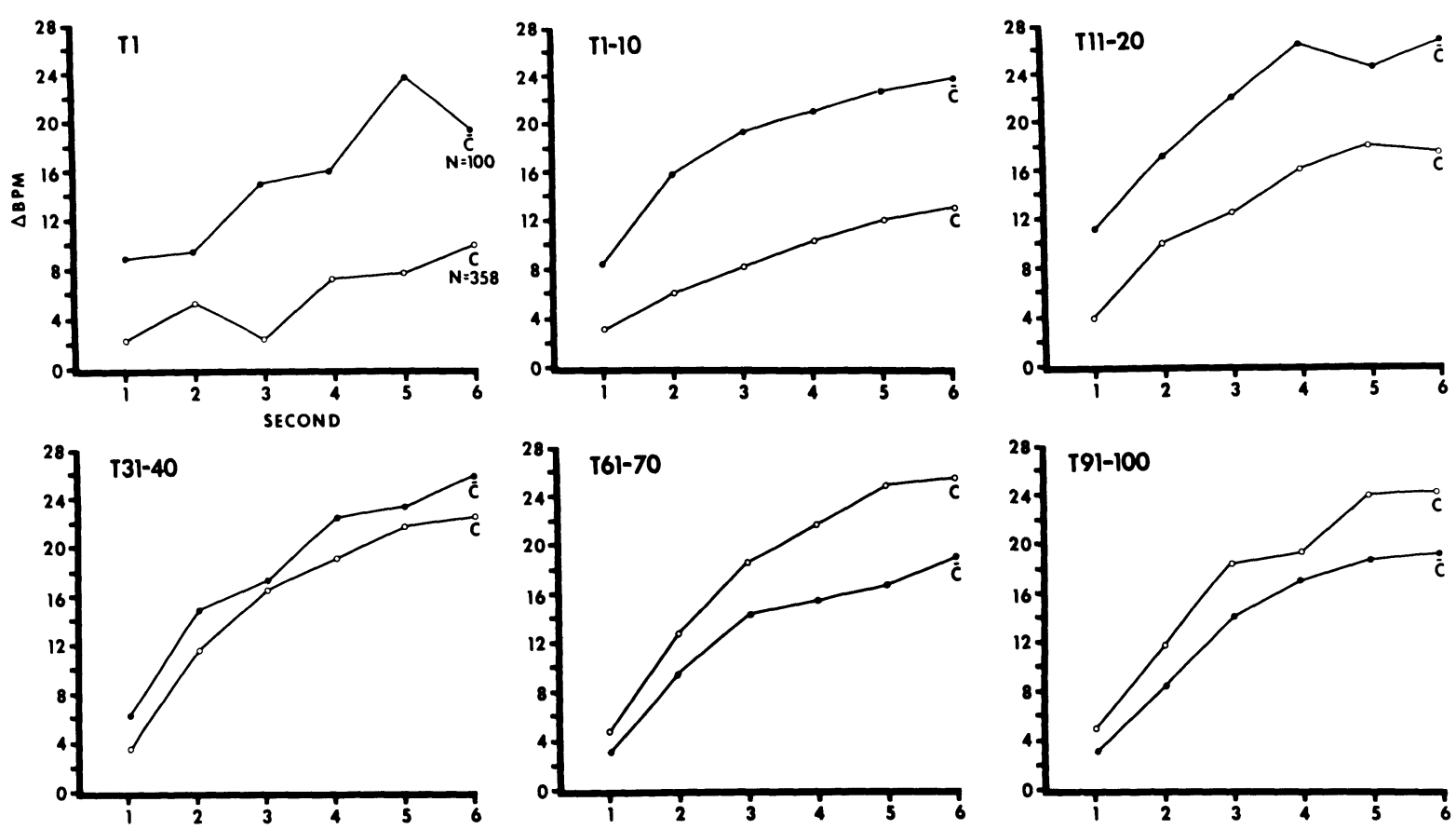

Figure 7. Mean response dynamics of the criterion $(C)$ and noncriterion $(\overline{\mathbf{C}})$ groups. These dynamics are shown for Trial 1 (T1) and for the 1st (T1-10), 2nd (T11-20), 4th (T31-40), 7th (T61-70), and 10th (T91-100) 10-trial blocks.

there were significant Groups by Trials interactions for the 2nd and 3rd sec, with $\bar{C}$ animals showing greater response development in this early portion of the stimulus period (Figure 7, second panel).

Similar analyses were performed for each $1-\mathrm{sec}$ interval of the 10-trial blocks. As a result of the crossover in response levels of the two groups (Figures 6 and 7), overall response differences for each second did not differ significantly. However, the Groups by Trials interactions were significant, with $p<.001$ for each of the six intervals. Thus, analysis of response dynamics merely confirms the larger responses of $\bar{C}$ animals early in training and their subsequent decline to a lower plateau. Still, the conditioned response was always monotonic cardioacceleration, with group differences being entirely accounted for by response magnitude.

In an effort to understand further the differences between $C$ and $\bar{C}$ animals, we compared them with respect to baseline heart rates and distribution over years and seasons. Chi-square analyses clearly indicated no differences in the relative proportions of $\mathrm{C}$ and $\bar{C}$ animals with respect to years or months. The baseline heart rates of the two groups are illustrated in Figure 8, and analysis of variance for the initial 10 trials indicated a significant Trials effect, $F(9,4104)=41.19, p<.001$, but no group difference or interaction. This implies that baseline rates do not account for the performance differences during these early trials. Analysis of variance of baselines for the 10-trial blocks also showed a signif- icant Trials effect, $F(9,4104)=152.80, p<.001$, but again, there was no significant group difference. There was, however, a significant Groups by Trials interaction, $F(9,4104)=12.10, p<.001$, reflecting a tendency in the $\mathrm{C}$ group toward slightly lower baseline rates as training progressed. Despite this, baseline rate would still not be a significant factor in accounting for the different acquisition patterns.

\section{DIFFERENCES WITHIN CRITERION AND NONCRITERION GROUPS}

Figure 5 includes information on the distribution of asymptotic trial blocks for the $\mathrm{C}$ group and the distribution of blocks where peak responses occurred for $\bar{C}$ animals. Since the range of these measures

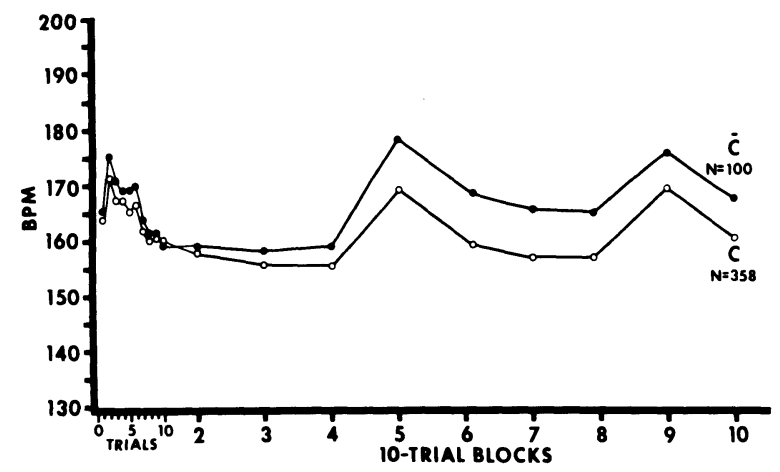

Figure 8. Mean baseline rates for the criterion (C) and noncriterion $(\overline{\mathbf{C}})$ groups. 
was broad within each group, we further subdivided them in hopes of gaining a more detailed understanding of acquisition patterns.

\section{Criterion Group}

For further analysis of the $\mathrm{C}$ group, we began by plotting mean learning curves for birds achieving criterion in each of the 10-trial blocks. An initial observation was that the curves for animals achieving criterion in Blocks 8,9 , or 10 were similar, and that they differed from the mean curves of the other $\mathrm{C}$ subgroups. Consequently, animals achieving criterion in these final training blocks were pooled (Figure 9, lower right). The resulting acquisition curve more closely resembles that of $\overline{\mathrm{C}}$ birds in having an early peak followed by a decline. It differs in having a late increase in response magnitude, and it is only because this increase exceeded the initial peak that criterion was attained. However, the late increases in response magnitude can also occur in certain $\bar{C}$ animals (Figure 10), and we thus suggest that animals achieving criterion in Blocks 8-10 are more appropriately considered as $\bar{C}$ animals in their acquisition pattern or at least as transitional between $\mathrm{C}$ and $\bar{C}$ groups.

The mean learning curves for groups achieving criterion within the first seven 10-trial blocks are also shown in Figure 9. First, we found no differ-
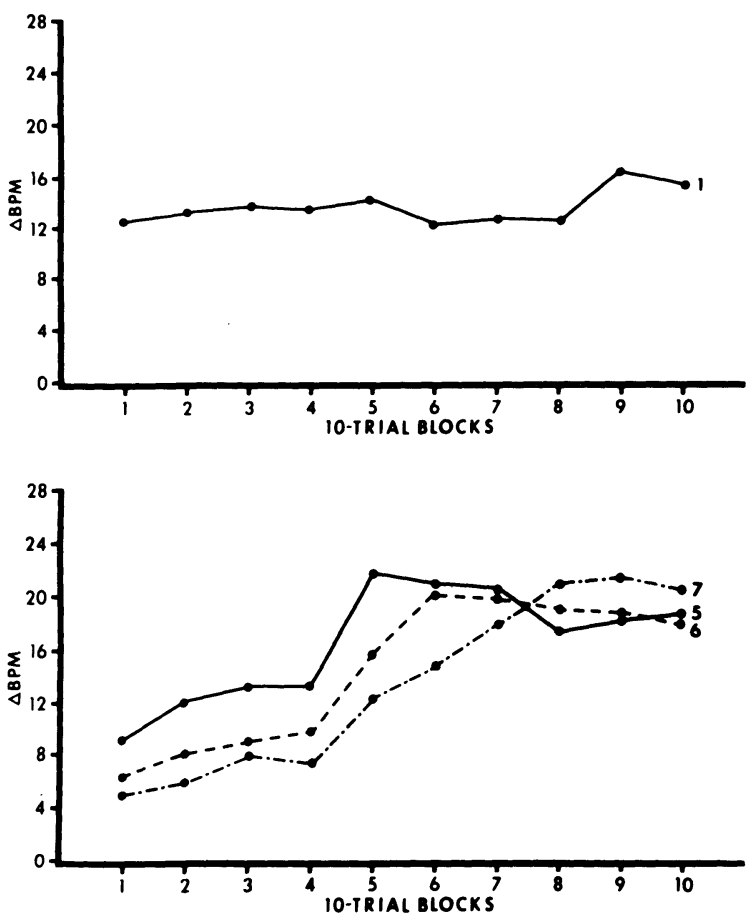

ences among any of these groups in their orienting responses, such that all were equivalent in response magnitude at the start of training. Next, consider the group reaching criterion in the initial block (Figure 9, upper left). In this group, responses increased within the first few trials and then maintained that level throughout training; a Scheffé test for multiple comparisons with $\alpha=.01$ confirmed that the 10-trial means were horizontally distributed.

The curves for birds achieving criterion in Blocks 2 , 3 , or 4 are shown at the upper right of Figure 9. These animals, as well as those achieving criterion in the first block, responded asymptotically by the end of the first training day, such that $45.6 \%$ of the total sample satisfied criterion within the first session. Inspection of the upper right panel of Figure 9 suggests that performance increases linearly to the asymptotic block and consequently, the slopes in this phase of training are successively lower for groups achieving criterion in Blocks 2, 3, and 4, respectively. Beyond the criterion block, performance remains stable for each group, and a comparison from the fifth block onward indicated neither significant group differences nor a Groups by Blocks interaction.

The lower left of Figure 9 presents the learning curves for animals reaching criterion on Blocks 5 , 6 , or 7 , that is, during the second session. These animals characteristically show slight but consistent
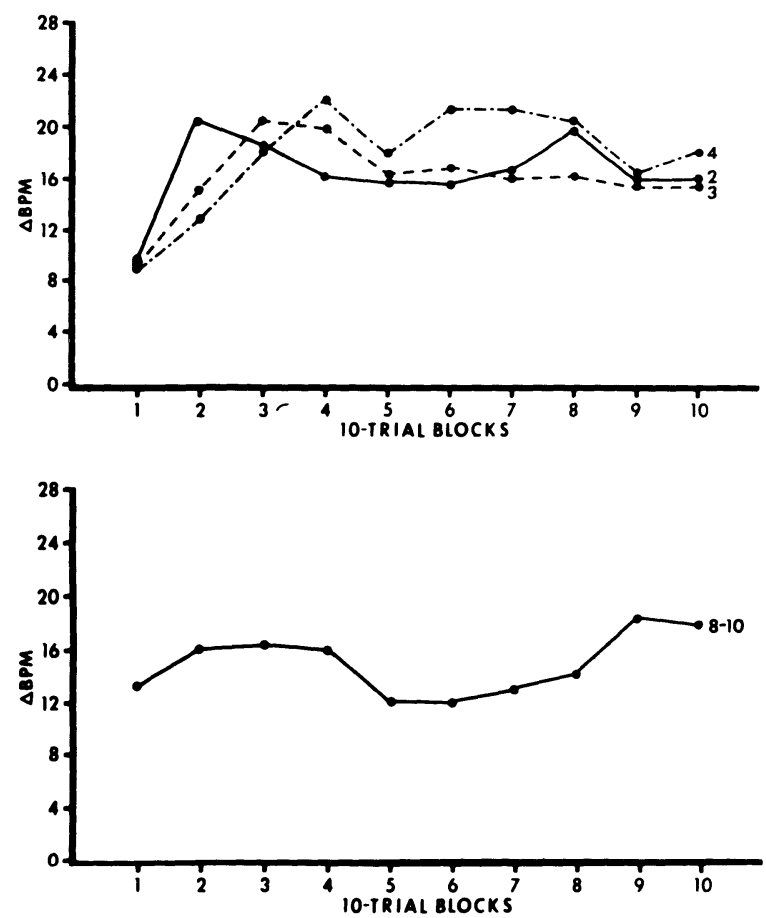

Figure 9. Mean acquisition curves of criterion (C) animals subdivided according to the 10-trial block in which asymptote was achieved (see Figure 5). The upper left shows the curve of birds reaching criterion in Blocks 2, 3, and 4. The lower left shows the acquisition curves of birds reaching criterion in Blocks 5, 6, and 7, and the lower right shows the pooled curve for animals achieving criterion in Blocks 8-10. 
response increases within the first 40-trial session, and their magnitude appears related to the rapidity with which criterion is attained in the second session. In this second session, there is a sharp slope increase for all three groups, and performance increases linearly to criterion. Beyond criterion, these groups respond at equivalent levels.

In summary, animals reaching criterion in Blocks 2-7 present a reasonably straightforward picture. Those achieving asymptotic level in the first session show a linear increase in performance to the criterion block and then maintain that response level. Those achieving asymptotic level in the second session show slower linear growth during the 1st day, but at the start of the second session the slope increases dramatically and criterion performance is approached linearly. Moreover, the asymptotic responses of these six groups do not differ significantly, implying that their eventual asymptote is not a function of the rate at which it is reached.

In contrast, animals achieving criterion during Block 1 or Blocks 8-10 seem different. Those reaching criterion late in training have a mean learning curve resembling that of $\bar{C}$ animals, and we interpret this as a transition between $C$ and $\bar{C}$ performances. The group attaining asymptote in Block 1 is puzzling, since there is no real growth or decline in performance over training; they simply maintain a mean performance level of 13.2 beats $/ \mathrm{min}$. One interpretation is that these birds are basically $\bar{C}$ animals that did not have sufficiently high response levels in the initial block to be classified as such. Consistent with this is the low asymptotic response level corresponding to the postpeak plateau of the $\bar{C}$ curve (Figure 6), and there are suggestive similarities between the curves for animals achieving criterion in the first block (Figure 9, upper left) and $\bar{C}$ animals with peak responses in this initial block (Figure 10, left).

\section{Noncriterion Group}

Figure 5 indicates that 100 animals never achieved criterion. To analyze this group further, we thus subdivided it with respect to the 10-trial block in which the peak response occurred. From Figure 5, it may be seen that $88 \%$ of $\bar{C}$ animals achieved this peak within the first four blocks, that is, during the first session. The remaining 12 birds had peak responses in Blocks 5-10, and because of their small number they were pooled to form a single group of late peak responders.

The mean learning curves for these five groups are shown in Figure 10. It should first be pointed out that the groups did not differ in their orienting responses and were therefore equivalent in response magnitude at the start of training. Regarding performance prior to the peak block, it tended to increase linearly to the peak. By definition, this statement cannot apply to animals achieving peak response in Blocks 1 or 2 , but it is suggested by the groups whose peaks occur in Blocks 3 and 4 . While animals attaining peak performance in Blocks 5-10 appear to violate this rule, it should be recalled that this is a composite group of small size.

Analysis of variance of peak responses indicated no differences among the five groups. Thus, like the $C$ group, the growth rate for $\bar{C}$ animals is not related to ultimate response magnitude. Beyond the peak block, all groups decline in response levels to a lower plateau (Figure 10). Analysis of these plateaus for Blocks 5-10, excluding the composite group, indicated stable response levels, since there was no significant Blocks effect or Groups by Blocks interaction. However, the groups did differ with respect to plateau level, $F(3,85)=3.55, \mathrm{p}<.05$, and these levels did not obviously relate to the peak block (Figure 10).

\section{PREDICTION OF CONDITIONING PERFORMANCE}

In this section, we attempt to apply the previous findings in two ways. First, we use the acquisition
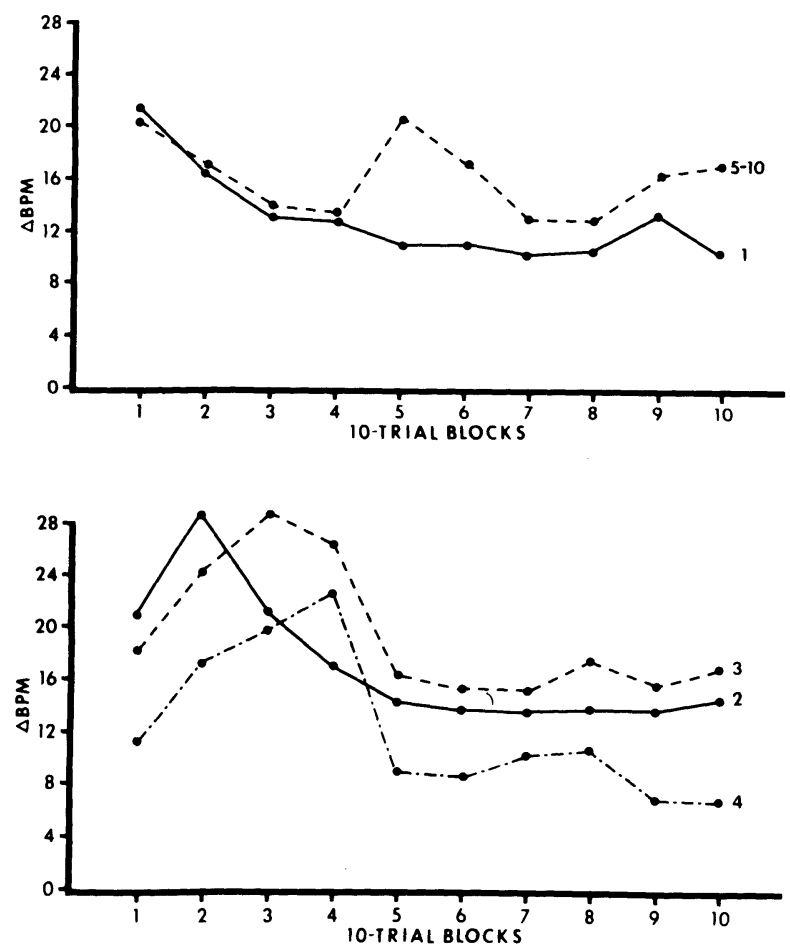

Figure 10. Mean acquisition curves of noncriterion $(\overline{\mathbf{C}})$ animals subdivided according to the 10 -trial block in which the peak response occurred (see Figure 5). The upper panel shows the curves of animals with peak responses in Block 1 and Blocks 5-10, inclusive. The bottom panel shows the curves of birds with peak responses in Blocks 2,3 , and 4 . 


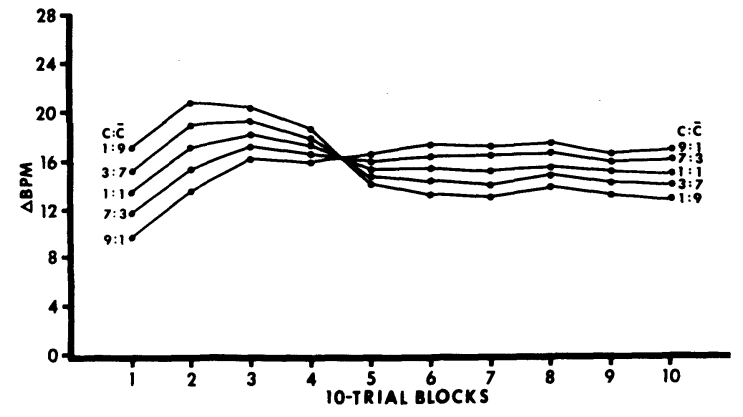

Figure 11. Theoretical mean acquisition curves for groups with different proportions of criterion $(C)$ and noncriterion $(\bar{C})$ animals, that is, with different $C: \bar{C}$ ratios. These were derived from the total sample $C$ and $\bar{C}$ curves shown in Figure 6.

pattern results to predict mean learning curves of independent samples not included in the previous analysis. Second, an effort is made to predict later performance from early training behavior, both on an individual and group basis.

\section{Prediction of Mean Learning Curves}

Since the $C$ and $\bar{C}$ learning curves differ so markedly (Figure 6), it is clear that the conformation of the mean curve for any given sample will depend upon its relative proportion of $C$ and $\bar{C}$ animals, the $C: \bar{C}$ ratio. Figure 11 shows a family of expected mean learning curves for different $C: \bar{C}$ ratios; these were derived from the curves of Figure 6, which represent ratios of 10:0 for the $C$ curve and 0:10 for the $\bar{C}$ curve. For example, the $1: 1$ curve represents a group with equal weighting of $C$ and $\bar{C}$ animals and is derived from an unweighted combination of the $C$ and $\bar{C}$ curves of Figure 6 . The mean curve for the entire sample (Figure 3 ) represents a ratio of $4: 1$, since $\cong 20 \%$ of the analyzed sample was classified as $\bar{C}$.

To assess the predictive value of the $\mathrm{C}: \overline{\mathrm{C}}$ ratio, we randomly selected three groups of 10 birds from our 1976 control population which was not included in the present analysis. The mean learning curve for each sample was calculated, as well as that for the pooled sample of 30 birds (Figure 12). The $C: \bar{C}$ ratio was then calculated as $1: 1,4: 1$, and $3: 2$ for Samples 1 , 2 , and 3, respectively; the ratio for the pooled sample of 30 was 19:11. The expected mean learning curves for the samples were derived from these ratios and are shown relative to the observed curves in Figure 12. In each case, the mean of the expected curve has been adjusted to equal that of the observed. The results of chi-square goodness-of-fit tests are shown in Table 2, and the first row indicates that, except for Sample 2, reasonable fits are obtained even without adjustment of the expected mean. Adjusting the means then improves prediction, and it is evident from Figure 12 that excellent fits are obtained for each sample. The second row of Table 2 gives goodness-of fit results for these comparisons, and the lower chi-square values indicate improved predictability for every case, most prominently for Sample 2 .

The above predictions were all based simply on the $C: \bar{C}$ ratio. Though the predicted curves were remarkably close to the observed, we attempted to improve the fit still further by taking into account the subdivisions of the $\mathrm{C}$ and $\overline{\mathrm{C}}$ groups. Recall that the $C$ group was subdivided with respect to the trial block where criterion was attained, and the $\bar{C}$ group was subdivided with respect to the peak response block. The independent samples were thus reevaluated to classify animals according to these subdivisions, and new expected mean learning curves were then derived from the curves shown in Figures 9 and 10. These are compared with the observed curves in Figure 12, where it is evident that predictability is only minimally improved (Table 2 ).

Given the success of the $C: \bar{C}$ ratio in predicting mean learning curves, a similar analysis was undertaken to predict response dynamics. However, for many of the trial blocks in each of the independent samples of 10 animals, chi-square evaluation indicated unacceptable fits, and therefore results are described for only the pooled sample of 30. Again, expected curves were calculated with and without adjusted means and on the basis of the $C: \bar{C}$ ratio with and without group subdivisions. None of these predictors provided a close fit to the observed orienting response. However, accurate prediction of response dynamics for the 10-trial blocks is obtained using the $\mathrm{C}: \overline{\mathrm{C}}$ ratio with the mean adjusted. As with the mean learning curve prediction, taking into account group subdivisions minimally improved goodness of fit. Thus, as anticipated from the excellent mean curve

Table 2

$\chi^{2}$ Goodness-of-Fit Results

\begin{tabular}{lcccc}
\hline & Sample 1 & Sample 2 & Sample 3 & Pooled \\
\hline Expected & 6.7 & 17.7 & 1.4 & 3.1 \\
& $.5<\mathrm{p}<.7$ & $.05<\mathrm{p}<.02$ & $.99<\mathrm{p}$ & $.98<\mathrm{p}<.95$ \\
Expected (mean adjusted) & 5.4 & 1.2 & 1.2 & .7 \\
Expected with Subgroupings (mean adjusted) & $.7<\mathrm{p}<.8$ & $.99<\mathrm{p}$ & $.99<\mathrm{p}$ & 2.3 \\
\hline
\end{tabular}



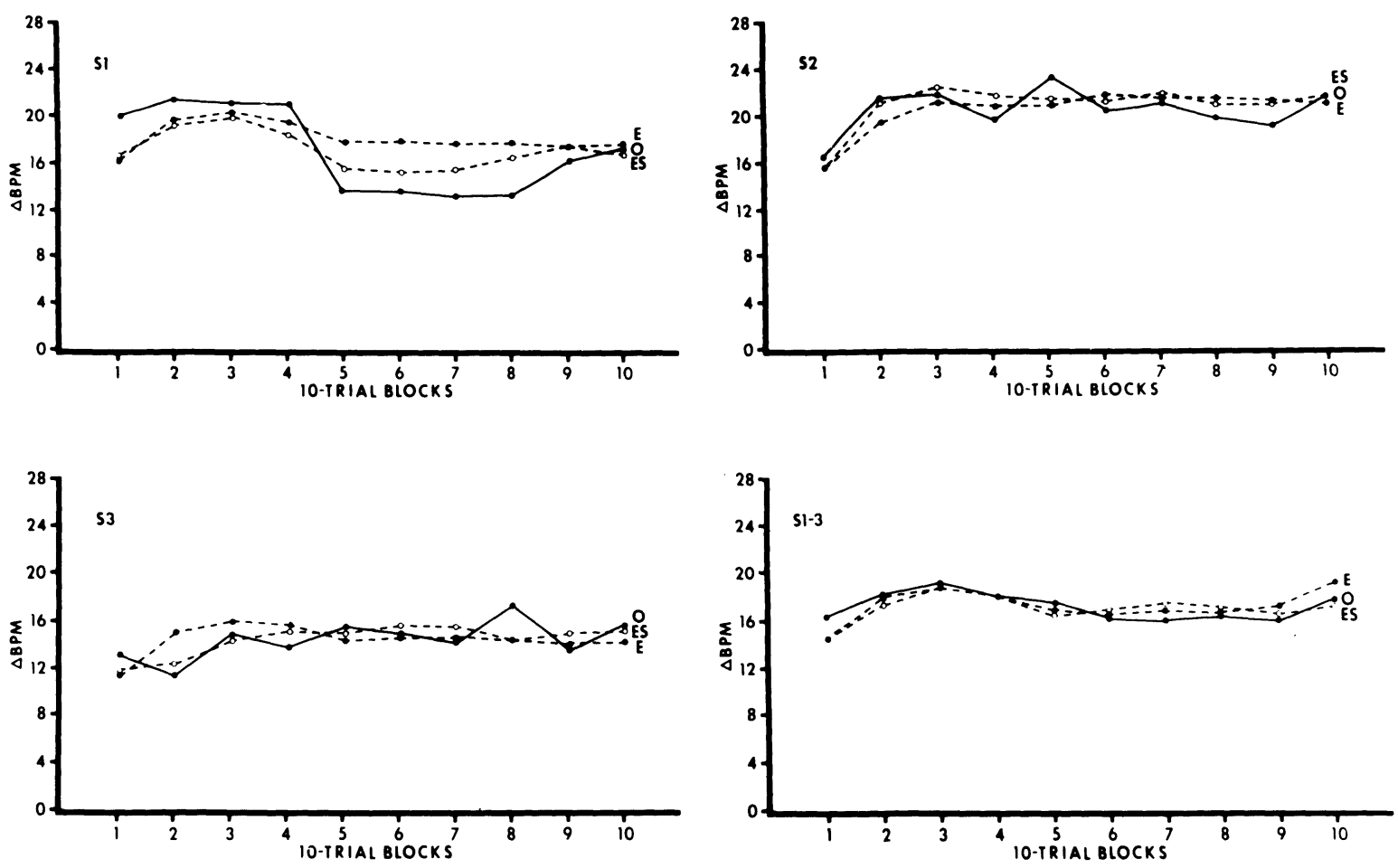

Figure 12. Comparisons of observed (O) mean acquisition curves from three independent samples (S1, S2, and S3) and from these samples pooled (S1-S3) with the expected (E) curve based on the $C: \bar{C}$ ratio of each of these samples. Also shown is the expected curve taking into account $\mathrm{C}$ and $\mathrm{C}$ subdivisions (ES). (See text for further explanation.)

prediction, response dynamics were predictable as well. However, this required a larger sample to obtain fits as close as those for the mean learning curves, and individual trial dynamics were difficult to predict reliably.

In summary, the $\mathrm{C}: \overline{\mathrm{C}}$ ratio is clearly of great predictive value, particularly for mean learning curve conformation. It is somewhat less accurate in predicting absolute response magnitudes and response dynamics, except for larger samples. Although considering $C$ and $\bar{C}$ subdivisions tends to improve predictability, the net gain is slight and not truly significant. Consequently, these results establish the $\mathrm{C}: \overline{\mathrm{C}}$ ratio as a reliable and simple predictor of group performance, and thus they tend to validate the distinction between these two classes of acquisition pattern. In contrast, our further subdivisions of the $\mathrm{C}$ and $\overline{\mathrm{C}}$ groups minimally improved predictability, suggesting that they represent a more subtle quantitative distinction among animals.

\section{Prediction of Acquisition Pattern}

We established above that mean learning curves can be accurately predicted for any given group merely by knowing the $\mathrm{C}: \overline{\mathrm{C}}$ ratio. Thus, if one had an a priori means of classifying birds as $\mathrm{C}$ or $\overline{\mathrm{C}}$ learners, then mean curves could be predicted without any knowledge of the conditioning behavior of the animals.
Model for individual acquisition pattern. Our initial effort in this regard was to explore rules that might allow one to classify individual animals. Since $\mathrm{C}$ and $\overline{\mathrm{C}}$ groups differ substantially in their orienting response magnitudes (Figures 6 and 7), an initial sorting rule was derived on the basis of this response. While we anticipated that birds with large orienting responses would be more likely to have a $\bar{C}$ acquisition pattern, no high response cutoff could be established that had any reasonable predictive value. However, the following rule provides a low response cutoff that segregates 70 birds, 65 of which are $C$ learners: If, for a given bird, $\left.R_{1}<\overline{(R}_{1}-\sigma_{R_{1}}\right)$, then it has the $C$ acquisition pattern; where $R_{1}$ is the bird's response on Trial $1, \bar{R}_{1}$ is the group mean response on Trial 1 , and $\sigma_{R_{1}}$ is the Trial 1 standard deviation. This rule relies only upon information from the initial light presentation, and it sorts $15.3 \%$ of the sample with a classification error of only $7 \%$.

Given this rather surprising success at predicting individual acquisition patterns from only the orienting response, we pursued this sorting approach further by taking into account behavior during the initial 10-trial block. Again, no high response cutoff could be derived that had any predictive value, but the following rule applied to the residual sample of 388 birds generated a low response cutoff that sorted an additional 57 birds, of which 55 had a $C$ acquisition pattern: If, for a given bird, $\left.R_{1-10}<\overline{(}_{1-10}-\sigma_{R_{1-10}}\right)$, 
then it has the $C$ acquisition pattern; where $R_{1-10}$ is the bird's mean response for Trial Block $1, \bar{R}_{1-10}^{1-10}$ is the group mean response for Trial Block 1, and $\sigma_{R_{1-10}}$ is the standard deviation for Trial Block 1 . Thus, utilizing the data from the first 10 trials, Rules 1 and 2 allow sorting of 127 birds, of which 120 have the $\mathrm{C}$ acquisition pattern; that is, $27.7 \%$ of the sample could be classified with an error of only $5.5 \%$.

It was possible to extend this analytic strategy by continuing to expand the sequence of sorting rules, each successive rule taking into account additional blocks of conditioning trials. In each case, the rules involved a low response cutoff, as above, and the application of each additional sorting rule successfully segregated an increasing proportion of the total sample. For example, a sequence of three rules, taking into account data through the second 10-trial block, classified $35.4 \%$ of the sample with $6.2 \%$ error; application of a five-rule sequence, taking into account six 10 -trial blocks, classified $70.7 \%$ of the total sample, with only $4 \%$ error.

The generality of these rules was evaluated by applying them to the independent sample of 30 animals, and this demonstrated their validity. For example, the five-rule sequence allowed sorting of $67 \%$ of the independent sample, comparing favorably with the $70.7 \%$ of the total sample. Of the 19 $\mathrm{C}$ birds in the independent sample, $17(89.5 \%)$ were correctly assigned, as compared with $85.5 \%$ in the total sample. This predictability is encouraging, particularly in view of the different $C: \bar{C}$ ratios for the large $(\cong 4: 1)$ and independent $(\cong 1.7: 1)$ samples.

Given the generality of this sorting procedure, it is worthwhile to consider its implications. First, a clear finding was its inability to classify $\bar{C}$ animals. Successful sorting always involved a low response cutoff that preferentially sorted $C$ animals. The failure to establish an effective high response cutoff reflected the substantial overlap between $C$ and $\bar{C}$ animals at the upper end of the response distribution. Thus, the sorting rules basically predict only the C acquisition pattern, leaving a residual group with an increasingly higher proportion of $\bar{C}$ animals, as the rules are sequentially applied. Second, though the sorting procedure is reasonably successful at predicting $C$ acquisition pattern, it could not classify animals with respect to acquisition subgroups within the $C$ and $\bar{C}$ groups. The sorted and residual groups at any point in the classification procedure distributed rather evenly over the $C$ and $\bar{C}$ subgroups, providing further evidence that the subdivisions within the $C$ and $\bar{C}$ groups may not be a reliable property of acquisition pattern.

In summary, sorting rules can be derived that are partially effective in classifying individual animals with respect to acquisition pattern, and this model is successful when applied to an independent sample.
However, it has limitations in not sorting $\overline{\mathrm{C}}$ birds, save by attrition, and in requiring the incorporation of too much training data for classification of an acceptable proportion of the animals. Furthermore, because this acquisition model is only partially successful in classifying individual animals with respect to acquisition pattern, it does not provide a sufficiently accurate prediction of the $C: \bar{C}$ ratio of a given group to allow reliable prediction of its mean learning curve.

Model for group acquisition pattern. In developing the above model, some properties of the data became evident that suggested that an effective model could be established for predicting the $C: \bar{C}$ ratio on a group basis. In certain respects, this is not as attractive as being able to assign individual birds to $\mathrm{C}$ and $\bar{C}$ groups on the basis of early training data. However, as shown below, it has the rather remarkable property of predicting the group $C: \bar{C}$ ratio on the basis of only orienting response data, and this, in turn, allows accurate prediction of the mean learning curve.

This group model is based upon a comparison of theoretical population values, derived from the large sample data presented here, with the orienting responses of the sample for which the $C: \bar{C}$ ratio is to be predicted. We begin by defining the following population parameters:

$\psi=$ the proportion of criterion animals in the theoretical population;

$\varrho=$ the proportion of animals in the theoretical population that is classified after applying the first sorting rule (see Model for Individual Acquisition Pattern); and

$\varepsilon=$ the proportion of sorted animals that are misclassified by the first sorting rule.

We next define the following parameters of the sample:

$\mathrm{N}=$ sample size;

$\mathrm{r}=$ the proportion of animals that is classified after applying the first sorting rule; and

$\mathrm{C}_{\mathrm{p}}=$ the predicted proportion of criterion animals in the sample.

It is $C_{p}$ that we wish to predict, since this enables one to calculate the $C: \bar{C}$ ratio, which, in turn, allows prediction of the mean learning curve of the sample. The group acquisition pattern model states that

$$
C_{p}=\psi+[r-(\varrho+\varepsilon)],
$$

and

$$
C: \bar{C}=\left(C_{p} N\right):\left(N-C_{p} N\right)
$$


From the large sample analysis, we estimate $\psi=.782$, since $78.2 \%$ of the population reached criterion. As described in the preceding section (Model for Individual Acquisition Pattern), the first sorting rule involves only the initial (orienting) responses to the light prior to any light-shock pairing. Applying this rule to the large sample sorted $15.3 \%$ of the animals, and therefore we estimate $\varrho=.153$. Of the 70 $(15.3 \%)$ sorted birds, $5(7.1 \%)$ were incorrectly identified as $C$ learners, and thus we estimate $\varepsilon=.071$. Substituting these values, the model can be expressed as

$$
\begin{aligned}
C_{p} & =.782+[r-(.153+.071)] \\
& =r+.558
\end{aligned}
$$

where empirically $.558 \leqslant C_{p} \leqslant 1$.

To evaluate the validity of this model, we then applied it to the independent sample of 30 birds. It may be recalled that this sample had a $C: \bar{C}$ ratio of $19: 11$, rather different from the $\cong 4: 1$ ratio of the large sample. To apply the above model, it is only necessary to determine the value of $r$ by use of the first sorting rule, and for the independent sample this sorted only 2 of the 30 birds to give $r=.067$. Substituting in the equation for the model, we then get $\mathrm{C}_{\mathrm{p}}=.625$. Therefore, the model predicts that the $C: \overline{\mathrm{C}}$ ratio is $18.75: 11.25$, which is within rounding error of the observed value of 19:11. To assure that this surprisingly accurate prediction was not fortuitous, we drew another independent sample $(n=25)$ from our 1976 data and again applied the model. The predicted $C: \bar{C}$ ratio for this new sample was 14.95:10.05, which compares quite favorably with the observed ratio of 16:9. Since we have already demonstrated (see Prediction of Mean Learning Curves) that mean learning curves can be predicted with considerable accuracy, given the $C: \bar{C}$ ratio, the success of the above model implies that such mean curves can now be accurately predicted given only a group's responses to the initial light presentation. That is, mean acquisition curves can be accurately generated having no a priori information on the group's learning behavior.

\section{CONCLUDING REMARKS}

The long-term nature of our program has allowed the analysis of heart-rate conditioning data from an unusually large sample of animals, and this has generally confirmed and extended our previous reports of the basic characteristics of this conditioned response in the pigeon. However, it has also generated some rather unexpected findings with respect to acquisition pattern and the prediction of conditioning performance in this experimental model system.
To begin with a review of basic performance characteristics, the absolute conditioned response levels were highly correlated with baseline heart rates, such that factors affecting baseline (e.g., season of training) also affect conditioned heart rates. However, these can be dissociated by taking the difference between baseline and conditioned response levels, and this difference score provides a measure largely free of many sources of variation. Analysis of the total sample data based upon this measure then confirmed that the orienting response is a small, monotonic cardioacceleration. With systematic light-shock pairings, the cardioacceleratory response rapidly increases in magnitude over the initial 10 trials. It then grows more slowly over subsequent trials, with the mean curve reaching an asymptote within 20-30 paired presentations. Analysis of the response dynamics indicated that they are invariably monotonic cardioacceleration, with a latency of less than $1 \mathrm{sec}$ and with peak levels in the final $2 \mathrm{sec}$ of the conditioned stimulus period. These findings are all confirmatory of earlier descriptions of heart-rate behavior in our model system (e.g., Cohen, 1974a).

The first major new finding derived from our analyses of individual animals with respect to criterion performance. Applying our criterion measure clearly partitioned the sample into two classes of acquisition pattern. The criterion, or $\mathrm{C}$ birds, have a monotonic, negatively accelerated mean learning curve resembling that of the total sample in conformation. In contrast, the noncriterion, or $\bar{C}$ birds, show an early response peak that then declines to a lower plateau. (This is not necessarily intended to imply a mutually exclusive dichotomy, since there may be a small proportion of animals having a transitional acquisition pattern.) Analysis of the response dynamics indicated that the orienting and conditioned responses for both $\mathrm{C}$ and $\overline{\mathrm{C}}$ birds were still monotonic cardioacceleration. Thus, acquisition pattern differences reflect differences in the magnitudes of these rate increases rather than distinct response conformations. While it is possible to subdivide further the two acquisition classes, these subgroupings do not seem to reflect reliable individual differences, in contrast to the basic $\mathrm{C}$ and $\overline{\mathrm{C}}$ classification.

Given two distinct acquisition patterns, the important question arises as to the basis for these differences. We have already excluded such straightforward factors as seasonal variation and baseline heart rates. Although sex differences cannot be definitively excluded, they seem unlikely in view of the 4:1 ratio of $\mathrm{C}$ to $\overline{\mathrm{C}}$ birds. Age differences also seem an unlikely explanation, since the animals were all within a 2-6 month range. However, we cannot exclude the possibility of some critical period occurring within this range. 
The major possibility we would suggest is an inherent difference in stimulus responsivity which could result from a variety of physiological factors. Individual differences of this nature in the context of conditioning have been recognized since the pioneering efforts of Pavlov (1927), and they have even been experimentally manipulated in more recent behavior genetics studies (Dykman, Murphree, \& Ackerman, 1966). Consistent with such an explanation is the larger orienting response of the noncriterion animals. This might be a manifestation of greater sensitization which could contribute to the early response peak of the noncriterion learners. To pursue this direction further it would seem important to assess sensitization as a possible intervening variable in determining acquisition pattern. If stimulus responsivity were established as an important factor in accounting for acquisition pattern differences, it would then be of value to determine the relative sympathetic and vagal contributions to the responses of $C$ and $\bar{C}$ learners as an initial effort in exploring physiological differences.

In any case, an important corollary of our demonstration of distinct classes of acquisition pattern is that the mean learning curve of a given group of animals will, to a large extent, be a function of the proportion of $C$ and $\bar{C}$ learners in that group. On this premise, we generated a family of theoretical learning curves for groups with different proportions of $C$ and $\bar{C}$ animals, and these proved highly successful in predicting the mean curves of independent samples consisting of as few as 10 animals. This further validated the distinction between the two acquisition patterns, and it suggests that sample differences in the $C: \bar{C}$ ratio account for a substantial amount of the variability in conditioning performance among samples.

Given the striking success of the $C: \bar{C}$ ratio in predicting mean learning curves, it would be of considerable significance if acquisition pattern could be determined for a given animal a priori, since this would allow prediction of mean learning curves with no knowledge of a given animal's conditioning behavior. Encouraging us in this direction was the finding that orienting responses of noncriterion animals were significantly larger than those of criterion birds. Unfortunately, on the basis of this orienting behavior we were able to predict successfully the acquisition class of only $15 \%$ of the population, and thus an individual model was only of limited utility. However, a group model was highly successful in that we were able to predict with great accuracy the proportion of criterion and noncriterion learners in a given sample, knowing only the orienting behavior of the group. This, in turn, allowed accurate prediction of the group's mean learning curve.
We find it rather remarkable that merely on the basis of responses to a single light presentation the mean acquisition characteristics of a group can be completely described. This may well have important implications for the nature of conditioning in our model system, since it demonstrates a tight coupling between nonassociative responses to the visual stimulus and subsequent associative responses developed to it. This suggests a strong underlying component of stimulus responsivity in the associative process, and it is, in fact, possible that a major element in conditioning involves preventing the extinction (or habituation) of nonassociative responses to the to-beconditioned stimulus. This, in turn, could imply that processes such as sensitization are a critical and integral feature of the conditioning process (Groves \& Thompson, 1970; Kandel, 1976).

\section{REFERENCE NOTES}

1. Cohen, D. H. Avian visual pathways involved in conditioned heart rate change to whole field illumination: Bilateral enucleation, interruption of centrifugal retinal control, and lesions of selected terminal fields of the optic tract. In preparation.

2. Gold, M. R., \& Cohen, D. H. Heart rate conditioning in pigeons immobilized with $\alpha$ Bungarotoxin. In preparation.

\section{REFERENCES}

Cohen, D. H. The hyperstriatal region of the avian forebrain: A lesion study of possible function, including its role in cardiac and respiratory conditioning. Journal of Comparative Neurology, 1967, 131, 559-570.

Cohen, D. H. Development of a vertebrate experimental model for cellular neurophysiologic studies of learning. Conditional Reflex, 1969, 4, 61-80.

Cohen, D. H. The neural pathways and informational flow mediating a conditioned autonomic response. In L. V. DiCara (Ed.), Limbic and autonomic nervous systems research. New York: Plenum Press, 1974. (a)

Conen, D. H. The effect of conditioned stimulus intensity on visually conditioned heart rate change in the pigeon: A sensitization mechanism. Journal of Comparative and Physiological Psychology, 1974, 87, 591-597. (b)

CoHen, D. H. Analysis of the final common path for heart rate conditioning. In P. A. Obrist, A. H. Black, J. Brener, \& L. V. DiCara (Eds.), Cardiovascular psychophysiology. Chicago: Aldine, 1974. (c)

CoHeN, D. H. Involvement of the avian amygdalar homologue (archistriatum posterior and mediale) in defensively conditioned heart rate change. Journal of Comparative Neurology, 1975, 160, 13-36.

CoHen, D. H. Current approaches to the mechanisms of memory. In J. L. McGaugh \& R. F. Thompson (Eds.), Handbook of neurobiology and behavior: Neurobiology of learning and memory. New York: Plenum Press, in press.

Cohen, D. H., \& Durkovic, R. G. Cardiac and respiratory conditioning, differentiation, and extinction in the pigeon. Journal of the Experimental Analysis of Behavior, 1966, 9, 681-688.

Cohen, D. H., \& Macdonald, R. L. Some variables affecting orienting and conditioned heart-rate responses in the pigeon. Journal of Comparative and Physiological Psychology, 1971, 74, 123-133.

Cohen, D. H., \& OBRist, P. A. Interactions between behavior 
and the cardiovascular system. Circulation Research, 1975, 37, 693-706.

Cohen, D. H., \& Pitts, L. H. Vagal and sympathetic components of conditioned cardioacceleration in the pigeon. Brain Research, $1968,9,15-31$.

Dykman, R. A., Murphree, O. D., \& Ackerman, P. T. Litter patterns in the offspring of nervous and stable dogs: II. Autonomic and motor conditioning. Journal of Nervous and Mental Disease, 1966, 141, 419-431.

Groves, P. M., \& Thompson, R. F. Habituation: A dual-process theory. Psychological Review, 1970, 77, 419-450.

Kandel, E. R. Cellular basis of behavior. New York: Freeman Press, 1976

LeONARD, R. B., \& Cohen, D. H. The peripheral unconditioned stimulus pathway in a model learning system involving defensively conditioned heart rate change in the pigeon. Journal of Comparative and Physiological Psychology, 1975, 89, 1083-1090.

Migani-Wall, S. A., Cabot, J. B., \& Cohen, D. H. Development of a neuromuscular blocking procedure for heart rate conditioning in immobilized pigeons. Psychophysiological Research, 1977, 14, 499-506.

Pavlov, I. P. Conditioned reflexes. London: Oxford University Press, 1927.

Tuge, H., \& YuEH, C. H. Functional compensation of the somatic and visceral components of the defensive conditioned reflexes after decerebration in young pigeons. Activitas Nervosa Superior, 1962, 4, 275-283.

(Received for publication December 12, 1977; revision accepted March 14, 1978.) 